\title{
Log expansions from combinatorial Dyson-Schwinger equations
}

\author{
Olaf Krüger ${ }^{1}$
}

Received: 29 July 2019 / Revised: 10 February 2020 / Accepted: 6 March 2020 / Published online: 7 April 2020 (c) The Author(s) 2020

\begin{abstract}
We give a precise connection between combinatorial Dyson-Schwinger equations and log expansions for Green's functions in quantum field theory. The latter are triangular power series in the coupling constant $\alpha$ and a logarithmic energy scale $L-\mathrm{a}$ reordering of terms as $G(\alpha, L)=1 \pm \sum_{j \geq 0} \alpha^{j} H_{j}(\alpha L)$ is the corresponding $\log$ expansion. In a first part of this paper, we derive the leading log order $H_{0}$ and the next-to ${ }^{(j)}$-leading log orders $H_{j}$ from the Callan-Symanzik equation. In particular, $H_{j}$ only depends on the $(j+1)$-loop $\beta$-function and anomalous dimensions. In two specific examples, our formulas reproduce the known expressions for the next-tonext-to-leading log approximation in the literature: for the photon propagator Green's function in quantum electrodynamics and in a toy model, where all Feynman graphs with vertex sub-divergences are neglected. In a second part of this work, we review the connection between the Callan-Symanzik equation and Dyson-Schwinger equations, i.e., fixed-point relations for the Green's functions. Combining the arguments, our work provides a derivation of the log expansions for Green's functions from the corresponding Dyson-Schwinger equations.
\end{abstract}

Keywords Log expansion · Green's functions · Dyson-Schwinger equations · Callan-Symanzik equation

Mathematics Subject Classification $81 \mathrm{~T} 18$

\section{Contents}

1 Introduction . . . . . . . . . . . . . . . . . . . . . 2176

1.1 Prologue: Green's functions in quantum field theory . . . . . . . . . . . . . . . . . . . 2176

1.2 Summary of the results . . . . . . . . . . . . . . . . . . . . . 2181

2 Results ................................. 2183

Olaf Krüger

olaf.krueger@univie.ac.at

1 Faculty of Physics, University of Vienna, Boltzmanngasse 5, 1090 Vienna, Austria 
2.1 The anomalous dimensions and $\beta$-function . . . . . . . . . . . . . . . . . . . . . 2184

2.2 Log expansions . . . . . . . . . . . . . . . . . . . . . . . . . . 2185

2.3 Examples: truncated propagator Green's functions and QED . . . . . . . . . . . . . . . . 2189

3 Mathematical background . . . . . . . . . . . . . . . . . . . . . . . . 2190

3.1 The grading of $\mathcal{H} \ldots \ldots \ldots \ldots \ldots$. . . . . . . . . . . . . . . . . 2190

3.2 Feynman rules from a Hopf algebraic point of view . . . . . . . . . . . . . . . . . . . . 2191

3.3 Graph insertion and Dyson-Schwinger equations . . . . . . . . . . . . . . . . . . 2193

3.4 Rederivation of the Callan-Symanzik equation . . . . . . . . . . . . . . . . . . . . . 2197

4 Multiple coupling constants . . . . . . . . . . . . . . . . . . . . . . . . . . . 2197

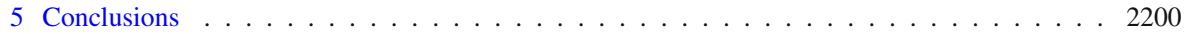

References. . . . . . . . . . . . . . . . . . . . . . . . 2201

\section{Introduction}

\subsection{Prologue: Green's functions in quantum field theory}

Quantum field theory (QFT) predicts probabilities for certain particle processes. For example, initialize an experiment, where a virtual photon decays into an electronpositron pair. One cannot predict whether and when the photon decays, and if it does, where the electron and positron will go in the end. However, one can put two detectors $D_{1}$ and $D_{2}$ somewhere and predict the probability that an electron enters $D_{1}$ and a positron enters $D_{2}$. This situation is totally different from deterministic classical mechanics, which predicts the exact time evolution for given initial conditions.

Such probabilities can be found in the following way: Each QFT is defined by a Lagrangian, which is a functional of the described particle fields. It consists of a kinetic part (terms that are quadratic in the fields) and interaction terms (of cubic or higher order in the fields). From all these, one can read off Feynman rules in a simple way. Now, a given particle process corresponds to a diagram (called Feynman graph) that translates via these rules to the so-called Feynman amplitude. Finally, the latter translates via the LSZ formula to a probability amplitude that squares to the actual probability. ${ }^{1}$

Consider, for example, the above experiment. The QFT that describes this process is quantum electrodynamics (QED). The corresponding Lagrangian is a functional of the photon field $A^{\mu}$, the electron $\psi$ and its antiparticle $\bar{\psi}$ (i.e., the positron). It contains one kinetic term for the photon and one for the electron and positron together. It also includes one interaction term, i.e., $g \bar{\psi} A^{\mu} \gamma_{\mu} \psi{ }^{2}$. Here, the coupling constant $g \ll 1$ is the electric charge of the electron and $\gamma_{\mu}$ is a Dirac matrix. Each process involving these particles translates to a graph that consists of edges and vertices: A photon corresponds to a wiggly line and a straight arrow line indicates an electron or positron (depending on the direction of the arrow). An interaction between these three particles is represented by a vertex. Table 1 shows all these basic ingredients of QED Feynman graphs. The virtual photon decay described above corresponds to the vertex graph in

\footnotetext{
1 This is similar to electrodynamics, where the $E$ field is an amplitude and only the intensity $I \propto E^{2}$ can be measured.

2 As a convenient toy model, we consider QED in Landau gauge. Here, another term in the Lagrangian vanishes [1] and the theory contains only one coupling constant. We discuss more general cases in Sect. 4.
} 
Table 1 Different types of edges and vertices in QED Feynman graphs

\begin{tabular}{lll}
\hline Electron and positron & Photon & Interaction vertex \\
\hline$\longrightarrow$ &
\end{tabular}

Table 1, and Feynman rules state that the respective Feynman amplitude is proportional to the electric charge $g$. It also depends on other factors, e.g., on the relative positions of the detectors.

Quantum mechanics now tells us that the obtained result is not exact. Indeed, one has to consider all possible ways in which the final state (an electron in $D_{1}$ and a positron in $D_{2}$ ) is achieved. For example, both particles could actually interchange another photon before entering the detectors. The respective Feynman diagram is the 1-loop graph

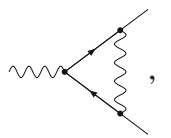

and one has to add the resulting Feynman amplitude to the previous one before computing the actual probability. Note that the latter amplitude is of order $g^{3}$, because by Feynman rules, each vertex constitutes one factor of $g$. Thus, the contribution to the overall probability is small compared to the initial one. All in all, one has to consider infinitely many Feynman graphs with more and more loops and vertices. However, their contributions to the final amplitude (the quantum corrections) become smaller and smaller. At some point, one may truncate the resulting Feynman amplitude when it is accurate enough.

There are two problems in this calculation: First, each loop diagram translates to a divergent integral via Feynman rules. In order to extract the correct quantum corrections, one has to regularize the integrals, i.e., to keep track of the different kinds of divergences. Then, one applies a renormalization scheme, i.e., one introduces 'counter terms' into the Lagrangian. These lead to additional Feynman graphs, whose amplitudes exactly cancel the divergences. In this way, the resulting Feynman amplitudes become finite. ${ }^{3}$ A second problem are infrared divergences that occur when the probability of a particle process is computed from the Feynman amplitude. We do not discuss these any further, because our work is focused on the computation of Feynman amplitudes.

After regularization and renormalization in a momentum subtraction scheme, each Feynman graph $\Gamma$ contributes a quantum correction $\phi_{R}(\Gamma)$ to the initial Feynman amplitude. This correction depends on the coupling constants $g_{k}$ and the scalar products $p_{i} \cdot p_{j}$ between the momenta $p_{i}^{\mu}$ of the particles that enter the process. Here, it is

\footnotetext{
3 Another way to think about regularization and renormalization is the following: The infinities are absorbed by the fields and coupling constants in the Lagrangian. In this way, they become unphysical, but the resulting probability amplitudes remain finite and are physically observable.
} 
very convenient to factor out an energy scale $S$ and to define dimensionless scattering angles as $\boldsymbol{\Theta}=\left\{p_{i} \cdot p_{j} / S\right\}$. Now, renormalization requires some sort of boundary condition. In our case, we assume that $\phi_{R}(\Gamma)$ is known for certain values of $S=S_{0}$ and $\boldsymbol{\Theta}=\boldsymbol{\Theta}_{0}$. One usually calls $S_{0}$ renormalization scale and $\left\{S_{0}, \boldsymbol{\Theta}_{0}\right\}$ renormalization point. ${ }^{4}$ Then, it turns out that $\phi_{R}(\Gamma)$ is a polynomial in the coupling constants $g_{k}$ and the $\operatorname{logarithm} L=\log \left(S / S_{0}\right)$ of the energy scale [2]. Hence, renormalized Feynman rules can be written as a linear map $\phi_{R}$ from the set of Feynman graphs to the polynomial algebra $\mathcal{A}\left[g_{k}, L\right]$. The dependence of $\phi_{R}(\Gamma)$ on the scattering angles $\boldsymbol{\Theta}$ and $\boldsymbol{\Theta}_{0}$ is hidden in the coefficients of the polynomial. In the following, we will make this more explicit.

First, $\phi_{R}$ is an algebra homomorphism - the domain of definition is the algebra $\mathcal{H}$ of all one-particle irreducible (1PI) Feynman loop graphs ${ }^{5}$ (of a given QFT) and their disjoint unions. The disjoint union is an associative product, and the empty graph $\mathbb{I}$ is the corresponding unit element. Furthermore, Feynman rules are normalized such that a 1PI Feynman graph $\Gamma$ contributes a change of $\phi_{R}(\Gamma)$ relative to the corresponding zero-loop Feynman amplitude. ${ }^{6}$ This implies that 1PI graphs without loops would map to 1 (the unit element in $\mathcal{A}$ ). Note that these are not in $\mathcal{H}$, but are identified with the empty graph $\mathbb{I}$. For example, the QED graphs in Table 1 would map to 1 by renormalized Feynman rules ${ }^{7}$ and the 1-loop graph in Eq. (1.1) maps to a term that is proportional to $g^{2}$ (and not $\propto g^{3}$ ). With this normalization, a one-particle reducible graph (1PR) corresponds to the disjoint union of its 1PI parts, e.g.,

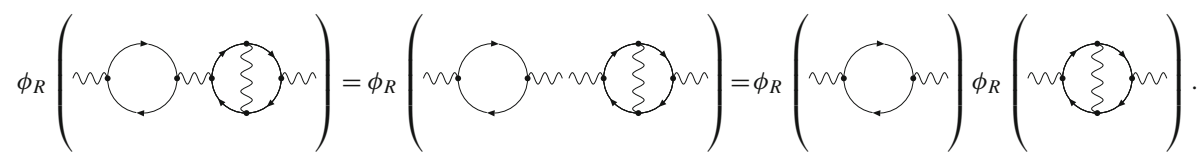

Secondly, the algebra $\mathcal{H}$ acquires a grading. In general, it is graded by the number of vertices of each kind in a graph. However, for QFTs with only one interaction term, it is more convenient to grade the algebra $\mathcal{H}$ by the number of loops,

$$
\mathcal{H}=\bigoplus_{k \geq 0} \mathcal{H}_{k}
$$

\footnotetext{
${ }^{4}$ In quantum chromodynamics (QCD), there are other kinds of boundary conditions. However, this does not alter the structure of $\phi_{R}(\Gamma)$ that is discussed in the following.

5 1PI Feynman graphs remain connected when one internal edge is removed. For example, 1 1PI, while $n$ ? $n$ is not. The latter is called one-particle reducible (1PR).

${ }^{6}$ In this way, $\phi_{R}(\Gamma)$ is a scalar, although the corresponding amplitude may be of a tensorial structure (e.g., it could be proportional to a Dirac matrix $\gamma_{\mu}$ ).

7 There is no problem that three different graphs are identified with the same element $\mathbb{I}$. All of them are mapped to 1 , so why distinguish?
} 
For example, $\mathcal{H}_{0}=\{\mathbb{I}\}$, the graph in Eq. (1.1) belongs to $\mathcal{H}_{1}$ and the disjoint union in Eq. (1.2) is an element in $\mathcal{H}_{3}$. In this case, there exists a redefinition of the coupling constant as $\alpha=g^{l}$, such that renormalized Feynman rules map each $k$-loop graph to a term proportional to $\alpha^{k}$. We show this explicitly in Sect. 3.1. For a vertex with three outgoing edges (e.g., QED), one finds $l=2$, whereas for a vertex with four outgoing edges (e.g., in $\phi^{4}$ theory), $l=1$. For simplicity, we state our results using this redefinition. The generalization to QFTs with more than one coupling constant is presented in Sect. 4.

All in all, renormalized Feynman rules are an algebra homomorphism

$$
\phi_{R}: \mathcal{H} \rightarrow \mathcal{A}[\alpha, L]
$$

and the quantum corrections of a certain particle process are found by applying $\phi_{R}$ to an infinite sum of elements in $\mathcal{H}$ (1PI graphs and disjoint unions thereof). This results in a series expansion in the coupling constant $\alpha$ and the logarithm $L$, where the dependence on the scattering angles is hidden in the coefficients. Moreover, the normalization of Feynman rules implies that $\phi_{R}$ evaluates Feynman graphs for scattering angles at the renormalization point, $\boldsymbol{\Theta} \rightarrow \boldsymbol{\Theta}_{0}$ (see [3]).

We will shortly explain the notion of Green's functions, using the above definition of renormalized Feynman rules. However, we first need some more notations: The residue $r$ of a Feynman graph is its external leg structure. For example, the graph in Eq. (1.1) has residue $r=n$ and those in Eq. (1.2) have residue $r=m$. Furthermore, its degree $|r|$ denotes the number of external edges-Feynman diagrams with $|r|=2$ are called 'propagator graphs' and those with $|r|>2$ 'vertex graphs'. Finally, we define $\operatorname{sgn}(r)$, which is +1 for vertex- and -1 for propagator graphs.

Now, in order to compute the exact Feynman amplitude of a certain particle process, one has to find the Green's functions of the respective QFT. These encode all necessary information about the full quantum corrections of all particle processes under consideration. For example, consider another QED experiment: a source $S$ emits an electron, which is absorbed at a later time by a detector $D$. The simplest way for the electron would be to go straight from $S$ to $D$. Then, the quantum corrections to the corresponding Feynman amplitude are obtained by applying renormalized Feynman rules to the sum of all Feynman diagrams with residue $r=\rightarrow$. We denote this sum by an ellipse. It is given by

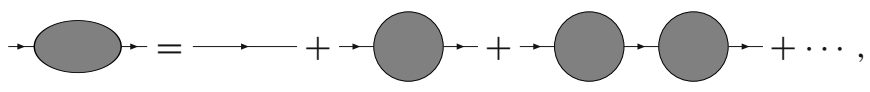

where the circle denotes the sum of all 1PI loop graphs. Thus, thanks to the geometric series,

$$
\phi_{R}(\rightarrow-)=\left[1-\phi_{R}(\rightarrow \bigcirc-)\right]^{-1} .
$$

This works for all propagator graphs. Hence, let

$$
X^{r}=\mathbb{I}-\sum 1 \text { PI loop graphs with residue } r, \quad|r|=2 \text {. }
$$


Then, the corresponding propagator Green's function $G^{r}$ is defined as $G^{r}(\alpha, L)=$ $\phi_{R}\left(X^{r}\right)$. It is the inverse of the quantum corrections to the respective 1-particle process. (The particle goes from $S$ to $D$.)

For interaction processes, the situation is similar, but there is no geometric series to compute. One simply defines

$$
X^{r}=\mathbb{I}+\sum 1 \text { PI loop graphs with residue } r, \quad|r|>2
$$

and calls $G^{r}$ with $G^{r}(\alpha, L)=\phi_{R}\left(X^{r}\right)$ the vertex Green's function of residue $r$. Knowing these functions allows to compute the correct Feynman amplitude of any interaction process that is described by the theory. Let us illustrate this again at the example of QED. First, consider the photon decay described at the beginning. The full quantum corrections are given by the Green's functions as follows:

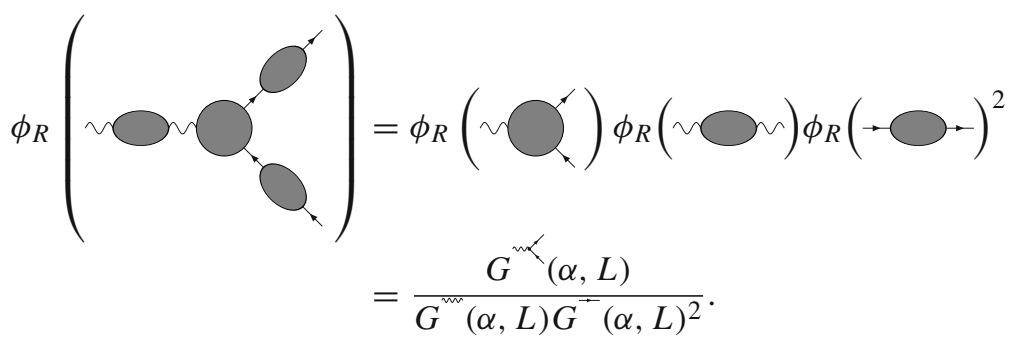

Here, the circle on the vertex denotes the sum of all 1PI graphs including the zero-loop graph, in contrast to the 'propagator circles'. Indeed, the argument of $\phi_{R}$ on the above lhs denotes the sum of all Feynman graphs with residue $r=n$. Secondly, consider a scattering between two electrons. The corresponding Feynman graphs have four external legs, and in this case, the zero-loop graph

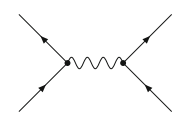

is not 1PI. But again, the full quantum corrections to the corresponding Feynman amplitude are given by the Green's functions:

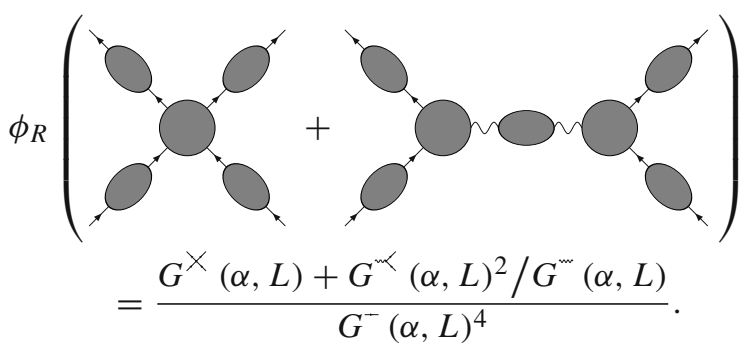

Note that the argument of $\phi_{R}$ is the sum of all Feynman graphs with residue before, the circle on the vertex with four outgoing edges denotes the sum of all corre- 
sponding 1PI graphs. However, it does not contain the respective zero-loop Feynman graph, because it does not exist. ${ }^{8}$

Let us summarize: One can compute the Feynman amplitude of any particle process when the Green's functions of the corresponding QFT are known. These are a power series in the coupling constant $\alpha$ and the logarithm of the energy scale $L=\log S / S_{0}$. Hence, $G^{r} \in \mathcal{A}[\alpha, L]$. But renormalized Feynman rules tell us slightly more, namely that a $k$-loop graph maps to a polynomial of degree smaller or equal than $k$ in $L$. We conclude that $G^{r}$ can be written as a triangular power series,

$$
G^{r}(\alpha, L)=\phi_{R}(r)+\operatorname{sgn}(r) \sum_{k \geq 1} \alpha^{k} G_{k}^{r}(L)
$$

where $G_{k}^{r}$ is a degree $k$ polynomial that encodes the contributions of all $k$-loop graphs to $G^{r} .{ }^{9}$ In particular, these polynomials vanish at the renormalization scale (for $L=$ $\left.\log S / S_{0}=0\right)$, such that

$$
G^{r}(\alpha, 0)=\phi_{R}(r)= \begin{cases}1, & \text { if the 1PI zero-loop graph } r \text { exists } \\ 0, & \text { else. }\end{cases}
$$

The Green's functions are infinite power series, and the best one can do is to approximate them perturbatively. One possibility is to truncate the sum in Eq. (1.9) at some loop order. Since $\alpha \ll 1$, this is a reasonable approach, at least for energies near the renormalization scale $S \sim S_{0}$. However, the perturbative computation breaks down at energies far away from $S_{0}$, where $L \gg 1$. Then, it is better to reorder the terms in Eq. (1.9) as follows:

$$
G^{r}(\alpha, L)=H^{r}(\alpha, \alpha L), \quad H^{r}(\alpha, z)=\phi_{R}(r)+\operatorname{sgn}(r) \sum_{j \geq 0} \alpha^{j} H_{j}^{r}(z) .
$$

This is called the log expansion for the Green's function $G^{r} . H_{0}^{r}$ is the leading $\log$ order (LLO), $H_{1}^{r}$ is the next-to-leading log order (NLLO) and in general, $H_{j}^{r}$ is the next-to ${ }^{(j)}$-leading log order $\left(\mathrm{N}^{(j)} \mathrm{LLO}\right)$. A truncation of the above sum, such that terms of order $\mathcal{O}\left(\alpha^{n+1}\right)$ are neglected, is called the next-to ${ }^{(n)}$-leading $\log \left(N^{(n)} L L\right)$ approximation of $H^{r}$. Perturbatively, this gives accurate results, as long as the functions $H_{j}^{r}$ are regular at $z=\alpha L$, which may well be the case for energies even far away from the renormalization scale.

\subsection{Summary of the results}

In this section, we outline our work and summarize the findings. For the explicit derivations, see the next sections.

\footnotetext{
${ }^{8}$ A comment on the notation: circles denote the sum of all 1PI graphs with the respective residue. For propagators, the zero-loop graph is excluded, while for vertices, it is included (if it exists).

9 The $\operatorname{sgn}(r)$ is a convention that is related to the different signs in the definitions of $X^{r}$ in Eqs. (1.5) and (1.6).
} 
In the first part of the paper, we derive the $\mathrm{N}^{(j)} \mathrm{LL}$ approximation of the Green's functions $H^{r}$ in Eq. (1.10) with $\phi_{R}(r)=1$. These are the propagator as well as the vertex Green's function that belongs to the interaction term in the Lagrangian. The general result is given in Eq. (2.18) in its most compact form. It is obtained by solving the Callan-Symanzik equation, which is a simple first-order partial differential equation for $G^{r}$ describing its dependence on the energy scale $L$; see Eq. (2.7). It turns out that $H_{j}^{r}$ only depends on the $(j+1)$-loop $\beta$-function and anomalous dimension. The latter are defined in Sect. 2.1 and only depend on the Feynman amplitudes of at most $(j+1)$-loop graphs.

In particle physics, it is long known how to solve the Callan-Symanzik equation in order to find the corresponding Green's function. See, for example, a standard textbook in quantum field theory (Peskin and Schroeder [4] in Section 12.3). There, the equations (12.76) and (12.79) show the two- and four-point Green's functions in a scalar $\phi^{4}$ theory. These formulas are specific examples of our Eq. (2.18), but written in a way that is more adapted to particle physics.

We find the explicit log expansions of the Green's functions by integrating and expanding the exponent in Eq. (2.18). This was not done before in the literature ${ }^{10}$ for the following reason: In QFT toy models and in many examples such as QED, socalled Ward-Takahashi identities (see, e.g., [5-7]) relate the Green's functions to the running coupling parameter. The latter can be found by solving the renormalization group equation (RGE), which is a linear first-order differential equation similar to the Callan-Symanzik equation. ${ }^{11}$ Now, the standard procedure in particle physics is to seek the log expansion of the running coupling parameter and to deduce the one for the Green's functions from there. In contrast, our approach is more general and can be directly used, even without Ward-Takahashi identities.

In order to check our formulas, we apply them to a class of QFT toy models, in which Feynman graphs with vertex sub-divergences are neglected; see Sect. 2.3. An example is the photon propagator Green's function $H^{m}$ in QED, thanks to the WardTakahashi identities. Here, we explicitly compute the NNLL approximations for the propagator Green's functions in Eq. (2.22) and give the precise connection to the log expansion of the running coupling parameter, see Eq. (2.21).

In the mathematical literature, there are two different direct approaches to obtain the $\mathrm{N}^{(j)}$ LL expansions in these toy models. This gives us the opportunity to compare our result to those derivations. In [8], the infinite sum $X^{r}$ in Eq. (1.5) is mapped to an element in the shuffle algebra of words. This paper describes an algorithm to filtrate a word into shuffles of letters and (anti-)symmetrized combinations of letters. The quasi-shuffle map $\theta$ operates as a symmetrizer, whereas the commutator [,] takes care of the anti-symmetrization. In the end, each term in the filtration belongs to a certain $\mathrm{N}^{(j)} \mathrm{LL}$ order of the corresponding Green's function $G^{r}$. Our results do not agree with the general findings of that work. The reason is a serious mistake in the filtration algorithm in [8]. More explicitly, the (anti-)symmetrization operators $\theta$ and [,] have been implemented self-consistently. However, combinations thereof lead to the wrong

\footnotetext{
10 At least, we are not aware of.

11 We introduce the running coupling parameter in Sect. 2.1. It is related to the $\beta$-function via the RGE, given in Eq. (2.8).
} 
filtration. The error can be traced back to the computation of a shuffle product of a letter $a$ with a commutator of two letters $b$ and $c$. In particular, the resulting expression depends on the order in which the steps are performed: replacing the commutator by $[b, c]=b c-c b$ before computing the shuffle product yields another result than doing it the other way around. ${ }^{12}$ Another approach to derive the log expansions in such toy models is given in [10] using chord diagrams. Our expression in Eq. (2.22) agrees with [10], and we give an explicit translation of the notation in Eq. (2.23).

In the second part of this paper, we review the connection between the CallanSymanzik equation and Dyson-Schwinger equations (DSEs) [11,12]. The latter are fixed-point relations for the infinite sums of Feynman graphs in Eqs. (1.5) and (1.6). The connection is established by extending $\mathcal{H}$ to the Hopf algebra of Feynman graphs $[13,14]$ and writing the DSEs using so-called insertion operators [15]; see Eq. (3.17).

Finally, we comment on possible extensions of our results. For the Green's functions $H^{r}$ in Eq. (1.10) with $\phi_{R}(r)=0$ (such as $H^{X}$ in QED), we are not aware of the existence of DSEs in the literature. However, if such fixed-point relations can be found in this case, it should also be possible to deduce a Callan-Symanzik-type equation for $H^{r}$. The latter can then be used to deduce the corresponding log expansion. On the other hand, there are DSEs for Green's functions in QFTs with more than one interaction term [14,16-18]—we generalize our results to such cases in Sect. 4.

The paper is organized as follows: In the next section, we derive the log expansions of the Green's functions $H^{r}$ with $\phi_{R}(r)=1$ for QFTs with only one interaction term in the Lagrangian. We also give the example of the propagator Green's functions in toy models that neglect Feynman graphs with vertex sub-divergences. The results are obtained from the Callan-Symanzik equation (2.7). In Sect. 3, we rederive that relation from Dyson-Schwinger equations for the infinite sums of Feynman graphs in Eqs. (1.5) and (1.6). There, we also review some known aspects about the combinatorics of Feynman graphs (we extend $\mathcal{H}$ to a Hopf algebra and rederive the essential properties of renormalized Feynman rules $\phi_{R}$ ). In Sect. 4, we generalize our results to QFTs with more than one interaction term in the Lagrangian and conclude in Sect. 5.

\section{Results}

In this section, we present the derivation of the log expansion $H^{r}$ in Eq. (1.10) with $\phi_{R}(r)=1$ from the Callan-Symanzik equation (2.7); see Sect. 2.2. The result contains the anomalous dimension and the $\beta$-function, which we introduce in Sect. 2.1. Finally, Sect. 2.3 gives a simple example of the log expansion for propagator Green's functions in QFT toy models, where all Feynman graphs with vertex sub-divergences are neglected.

\footnotetext{
12 This mistake does not affect the LL approximation, which was computed in general in [9], using the shuffle algebra of words. Here, our results agree; see Eq. (2.19) and the equations after (49) in [9].
} 


\subsection{The anomalous dimensions and $\beta$-function}

For each vertex residue $r=v$, define

$$
X_{\mathrm{amp}}^{v}=\frac{X^{v}}{\prod_{e \sim v} \sqrt{X^{e}}} .
$$

Here, $e \sim v$ means that the edge $e$ is incident to the vertex $v$. The square root must be expanded in a Taylor series, similar to the geometric series of propagator Feynman graphs. For example, in QED:

$$
X_{\mathrm{amp}}^{\mathrm{\alpha}}=\frac{X^{\mathrm{\alpha}}}{\sqrt{X^{\omega}} X^{-}}=\mathrm{C}^{\mathrm{x}}, X_{\mathrm{amp}}^{\mathrm{x}}=\frac{X^{x}}{\left(X^{-}\right)^{2}}=
$$

The square roots are graphically represented by half ellipses. Since renormalized Feynman rules $\phi_{R}$ are an algebra homomorphism, one can easily evaluate these infinite sums of Feynman graphs:

$$
G_{\mathrm{amp}}^{v}=\phi_{R}\left(X_{\mathrm{amp}}^{v}\right)=\frac{G^{v}}{\prod_{e \sim v} \sqrt{G^{e}}} .
$$

In physics literature, $G_{\mathrm{amp}}^{v}$ is often referred to as the amputated Green's function for the vertex $v$. In more mathematical literature, these functions are called the invariant charges.

Let us shortly motivate the above definition by showing how the amputated Green's functions appear in physics computations. For example, consider any particle interaction process that is described by the QFT. Let $v$ be the residue of the respective Feynman graphs. Then, one can easily show that the corresponding Feynman amplitude (including all quantum corrections) is given by $\prod_{e \sim v}\left(G^{e}\right)^{-1 / 2}$ times a polynomial of the amputated Green's functions. For example, the right-hand sides of Eqs. (1.7) and (1.8) read

$$
\frac{G_{\mathrm{amp}}^{\mathrm{w}}}{\sqrt{G^{\mathrm{w}}} G^{-}} \text {and } \frac{G_{\mathrm{amp}}^{\times}+\left(G_{\mathrm{amp}}^{\mathrm{\alpha}}\right)^{2}}{\left(G^{-}\right)^{2}} \text {. }
$$

Secondly, it is common in particle physics to redefine the coupling constants of the theory as $\tilde{g}_{k}=g_{k} G_{\mathrm{amp}}^{v_{k}}$. This makes them dependent on the energy scale and has the advantage that a perturbative expansion for the Green's functions in terms of $\tilde{g}_{k}$ and the logarithmic energy scale $L$ may behave much better. This is an alternative way to approximate the Green's functions accurately without using the log expansion. The $\tilde{g}_{k}$ are called the running coupling parameters. In our case, there is only one coupling constant and we redefined it such that $\alpha=g^{l}$ counts the number of loops in a Feynman graph. Hence, we must also redefine the respective invariant charge as ${ }^{13}$

$$
X_{Q}=\left(X_{\mathrm{amp}}^{v}\right)^{l}, \quad Q(\alpha, L)=\phi_{R}\left(X_{Q}\right)=G_{\mathrm{amp}}^{v}(\alpha, L)^{l}
$$

13 Here, $v$ is the vertex residue that corresponds to the interaction term in the Lagrangian. 
Then, the running coupling parameter is $\tilde{\alpha}=\alpha Q$. At the renormalization scale $(L=$ $0)$, one has $\tilde{\alpha}=\alpha$, because $Q(\alpha, 0)=1$.

Now, the anomalous dimension $\gamma^{r}$ is defined as the negative of the $L$-linear part of the Green's function $G^{r}$,

$$
\gamma^{r}(\alpha)=\sum_{k \geq 0} \gamma_{k}^{r} \alpha^{k+1}=-\left.\frac{\partial G^{r}(\alpha, L)}{\partial L}\right|_{L=0}
$$

Similarly, the $\beta$-function ${ }^{14}$ is the $L$-linear part of the invariant charge,

$$
\beta(\alpha)=\sum_{k \geq 0} \beta_{k} \alpha^{k+1}=\left.\frac{\partial Q(\alpha, L)}{\partial L}\right|_{L=0}=l\left(\frac{1}{2} \sum_{e \sim v} \gamma^{e}(\alpha)-\gamma^{v}(\alpha)\right) .
$$

The latter identity follows from Eq. (2.2): the $\beta$-function is a linear combination of the various anomalous dimensions. For example, $l=2$ in QED:

$$
Q(\alpha, L)=G_{\mathrm{amp}}^{m \alpha}(\alpha, L)^{2}, \quad \beta=2 \gamma^{-}+\gamma^{m}-2 \gamma^{m<} \text {. }
$$

Two remarks are in order: First, the notion of an anomalous dimension $\gamma^{r}$ for vertex-type residues is unusual in particle physics. Indeed, it is redundant, since $\gamma^{v}$ can be expressed by the $\beta$-function and the various propagator anomalous dimensions $\gamma^{e}$ via Eq. (2.5). In our case, however, the definition in Eq. (2.4) including vertextype residues is very convenient because the formulas for the log expansions of the Green's functions $H^{r}$ generalize to all kinds of residues. Secondly, the minus sign in Eq. (2.4) is chosen such that it cancels the sign in Eq. (1.5) for propagator-type residues $r=e$. In this case, the anomalous dimension $\gamma^{e}$ is given by the $L$-linear part of the Feynman amplitude that corresponds to the sum of all respective 1PI propagator graphs.

The functions $\gamma^{r}$ and $\beta$ can be computed perturbatively. Neglecting terms of order $\mathcal{O}\left(\alpha^{n+1}\right)$ in Eqs. (2.4) and (2.5) gives the so-called $n$-loop anomalous dimensions and $n$-loop $\beta$-function. These approximations can be obtained by only evaluating Feynman graphs with at most $n$ loops. It turns out that the $\mathrm{N}^{(n)} \mathrm{LL}$ approximation of the Green's functions $H^{r}$ only depend on the $(n+1)$-loop anomalous dimensions $\gamma^{r}$ and $\beta$-function. We show this explicitly in the next section.

\subsection{Log expansions}

We start with the following formula for the Green's functions:

$$
\frac{\partial \log G^{r}}{\partial L}=-\gamma^{r}(\alpha Q)
$$

A detailed derivation from combinatorial Dyson-Schwinger equations is collected in Sect. 3 (for a short proof, see Sect. 3.4). Taking a linear combination of the above rela-

\footnotetext{
14 In our case, there is only one $\beta$-function.
} 
tions for different residues $r$ implies an ordinary differential equation for the invariant charge, i.e.,

$$
\frac{\partial \log Q}{\partial L}=\beta(\alpha Q)
$$

Together with the initial conditions,

$$
G^{r}(\alpha, 0)=\phi_{R}(r)=1, \quad Q(\alpha, 0)=1,
$$

Equations (2.7) and (2.8) determine the full $L$-dependence of the Green's functions $G^{r 15}$.

In particle physics, the above relations are usually written in terms of the $(L$ dependent) running coupling parameter $\tilde{\alpha}=\alpha Q$. Then, Eq. (2.8) corresponds to the renormalization group equation (RGE) ${ }^{16}$ and Eq. (2.7) represents the CallanSymanzik equation for the Green's function $\tilde{G}^{r}$ with $\tilde{G}^{r}(\tilde{\alpha}, L)=G^{r}(\alpha, L)$ :

$$
\frac{\partial \tilde{\alpha}}{\partial L}=\tilde{\alpha} \beta(\tilde{\alpha}), \quad\left(\frac{\partial}{\partial L}+\tilde{\alpha} \beta(\tilde{\alpha}) \frac{\partial}{\partial \tilde{\alpha}}+\gamma^{r}(\tilde{\alpha})\right) \tilde{G}^{r}(\tilde{\alpha}, L)=0 .
$$

The derivation of the log expansions for the Green's functions requires another differential equation for the invariant charge $Q$. The latter can be found as follows: Integrating Eq. (2.8) via separation of variables and using the above definition of the running coupling parameter leads to

$$
\int_{\alpha}^{\tilde{\alpha}} \frac{\mathrm{d} x}{x \beta(x)}=L
$$

Here, the integration constant is well chosen such that $\tilde{\alpha}=\alpha$ for $L=0$. Since we cannot solve this for $Q$, it is more convenient to take the derivative with respect to $\alpha$. This results in

$$
1+\alpha \frac{\partial \log Q}{\partial \alpha}=\frac{\beta(\alpha Q)}{\beta(\alpha)} .
$$

With these considerations, we can now give the main equations that determine the log expansions for the Green's functions. Here, it is convenient to define a log expansion for the invariant charge as well, ${ }^{17}$

$$
Q(\alpha, L)=R(\alpha, \alpha L), \quad R(\alpha, z)=R_{0}(z)-\sum_{j \geq 1} \alpha^{j} R_{j}(z)
$$

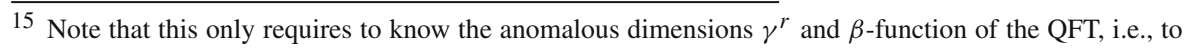
say the $L$-linear parts of the Green's functions.

16 The usual definition of the $\beta$-function in the literature slightly differs from ours:

$$
\beta_{\text {lit }}(\tilde{\alpha})=\tilde{\alpha} \beta(\tilde{\alpha}) .
$$

For example, the QED 1-loop $\beta$-function is $\beta_{\text {lit }}(\tilde{\alpha})=\beta_{0} \tilde{\alpha}^{2}$. The notations in this paper are consistent, such that our 1-loop $\beta$-function is linear in the (loop counting) coupling parameter.

17 Accordingly, the log expansion for the running coupling is given by $\tilde{\alpha}(\alpha, z)=\alpha R(\alpha, z)$. 
A change of variables in Eqs. (2.7-2.10) finally results in

$$
\begin{aligned}
& \frac{\partial \log H^{r}}{\partial z}=-\frac{\gamma^{r}(\alpha R)}{\alpha}, \quad H^{r}(\alpha, 0)=1, \\
& \frac{\partial \log R}{\partial z}=\frac{\beta(\alpha R)}{\alpha}, \quad R(\alpha, 0)=1, \\
& 1+\alpha \frac{\partial \log R}{\partial \alpha}-\frac{\beta(\alpha R)}{\beta(\alpha)}\left(1-\frac{z \beta(\alpha)}{\alpha}\right)=0 .
\end{aligned}
$$

Now, one uses Eqs. (2.13) and (2.14) in order to find the $\mathrm{N}^{(n)}$ LL approximation of the invariant charge. ${ }^{18}$ The obtained result can then be used in Eq. (2.12) to deduce the $\mathrm{N}^{(n)}$ LL approximation of the Green's functions $H^{r}$.

The first step is simply achieved by a Taylor series expansion of Eq. (2.14) in $\alpha$. The 0 th order reads

$$
R_{0}(z)=\frac{1}{1-\beta_{0} z},
$$

which gives the LLO of the invariant charge. Now, consider all terms of order $\alpha^{n}$ in Eq. (2.14) with $n \geq 1$ and note that they do not contain any of the functions $R_{N}$ with $N>n$. Hence, collecting all terms that involve $R_{n}$, one finds

$$
\frac{1-n}{R_{0}(z)} R_{n}(z)=\cdots,
$$

where the rhs only consists of the functions $R_{0}, \ldots, R_{n-1}$. This is a nice recursive formula for the various log orders of the invariant charge, except for $n=1$. Two remarks are in order: First, the $\alpha$-linear terms in Eq. (2.14) vanish completely, because an $\alpha$-derivative was taken in order to obtain Eq. (2.10). However, $R_{1}$ can be found from the $\alpha$-linear part of Eq. (2.13), which reads

$$
\frac{\mathrm{d} R_{1}}{\mathrm{~d} z}=2 \beta_{0} R_{0} R_{1}-\beta_{1} R_{0}^{3} .
$$

The solution of this ordinary first-order differential equation with the respective initial condition $R_{1}(0)=0$ is given by

$$
R_{1}(z)=-\frac{\beta_{1}}{\beta_{0}} R_{0}(z)^{2} \log R_{0}(z) .
$$

Secondly, an explicit form of the recursive relation for $R_{n}$ is not required. This is because Eq. (2.14) with cleared fractions can be given directly to a computer algebra program in order to obtain the various log orders. For example, the $\alpha^{2}$ order in Eq. (2.14) reads

\footnotetext{
18 This is equivalent to solving the RGE and implies a log expansion for the running coupling parameter $\tilde{\alpha}$; see, e.g., [19].
} 


$$
R_{2}(z)=R_{0}(z)^{3}\left(-\beta_{2} z+\frac{\beta_{1}^{2}}{\beta_{0}^{2}}\left(\beta_{0} z-\log R_{0}(z)-\log ^{2} R_{0}(z)\right)\right)
$$

when Eqs. (2.15) and (2.16) are used.

The second step (the derivation of the log expansion for the Green's functions $H^{r}$ ) is a bit more involved, because one has to integrate $\gamma^{r}(\alpha R)$ with respect to $z$. Indeed, from Eq. (2.12), ${ }^{19}$

$$
H^{r}(\alpha, z)=\exp \left(-\int_{0}^{z} \frac{\gamma^{r}\left(\alpha R\left(\alpha, z^{\prime}\right)\right)}{\alpha} \mathrm{d} z^{\prime}\right)
$$

In order to obtain the $\mathrm{N}^{(n)} \mathrm{LL}$ approximation of $H^{r}$, one needs to expand the exponent up to order $\alpha^{n+1}$. As promised earlier, this requires knowledge of the $(n+1)$-loop anomalous dimension $\gamma^{r}$ and $\beta$-function.

For example, the LL approximation requires to compute the nominator in the exponent up to first order in $\alpha$. Using the one-loop anomalous dimension (see Eq. 2.4) and the explicit form of $R_{0}$ in Eq. (2.15), we find

$$
H^{r}(\alpha, z)=\exp \left(-\int_{0}^{z} \gamma_{0}^{r} R_{0}\left(z^{\prime}\right) \mathrm{d} z^{\prime}\right)+\mathcal{O}(\alpha)=R_{0}(z)^{-\gamma_{0}^{r} / \beta_{0}}+\mathcal{O}(\alpha)
$$

This expression agrees with the result already obtained in [9] using the shuffle algebra of words.

We close this section by contentedly giving the NNLL approximation. Here, the exponent in Eq. (2.18) must be computed up to third order in $\alpha$. This requires knowledge of the three-loop anomalous dimension and the invariant charge up to NNLL order $\left(R_{0}, R_{1}\right.$ and $\left.R_{2}\right)$. The integration is technical, but can always be done with the help of a computer algebra program. We used FORM [21] to obtain

$$
\begin{aligned}
H^{r}(\alpha, z)= & R_{0}(z)^{-\gamma_{0}^{r} / \beta_{0}}\left[1+\frac{\alpha R_{0}(z)}{\beta_{0}^{2}}\left(\gamma_{0}^{r} \beta_{1}\left(1-\log R_{0}(z)\right)-\gamma_{1}^{r} \beta_{0}\right)\right. \\
& +\frac{\alpha^{2} R_{0}(z)^{2}}{2 \beta_{0}^{4}} \times\left(\left(\beta_{1} \gamma_{0}^{r}-\beta_{0} \gamma_{1}^{r}\right)^{2}+\beta_{0} \beta_{1}^{2} \gamma_{0}^{r}-\beta_{0}^{2} \beta_{2} \gamma_{0}^{r}+\beta_{0}^{2} \beta_{1} \gamma_{1}^{r}-\beta_{0}^{3} \gamma_{2}^{r}\right. \\
& +2\left(\beta_{0}^{2} \beta_{2} \gamma_{0}^{r}-\beta_{0} \beta_{1}^{2} \gamma_{0}^{r}\right) R_{0}(z)^{-1} \\
& -2\left(\beta_{1}^{2}\left(\gamma_{0}^{r}\right)^{2}-\beta_{0} \beta_{1} \gamma_{0}^{r} \gamma_{1}^{r}+\beta_{0}^{2} \beta_{1} \gamma_{1}^{r}\right) \log R_{0}(z) \\
& \left.\left.+\left(\beta_{1}^{2}\left(\gamma_{0}^{r}\right)^{2}-\beta_{0} \beta_{1}^{2} \gamma_{0}^{r}\right) \log ^{2} R_{0}(z)\right)+\mathcal{O}\left(\alpha^{3}\right)\right]
\end{aligned}
$$

\footnotetext{
19 In [20], the author discussed the behavior of the Green's functions at fixed points. In this case, the invariant charge evaluates to unity, $R(\alpha, z)=1$ and Eq. (2.18) reproduces the known results.
} 


\subsection{Examples: truncated propagator Green's functions and QED}

Let us give a short example of the above formula (2.20). In QED, the Ward-Takahashi identities state that the Green's functions for the fermion propagator $G^{-}$and the 3point vertex $G^{n<}$ coincide [22]. Therefore, the invariant charge in Eq. (2.3) is given by the inverse of the photon propagator Green's function. Furthermore, the $\beta$-function equals the respective anomalous dimension, see Eq. (2.6):

$$
Q=\frac{1}{G^{m}} \text { and } \beta=\gamma^{m} \text {. }
$$

The above relation is a special case of a broader class of QFT toy models that describe only one particle (edge-type $e$ in Feynman graphs). In such models, the invariant charge and $\beta$-function are given by

$$
Q=\left(G^{e}\right)^{-s} \text { and } \beta=s \gamma^{e}
$$

for integers $s$. Examples are $s=1$ in QED, as well as $s=2(s=3)$ for a truncated Yukawa theory ( $\phi^{3}$ theory), where all vertex divergences are neglected. In all these cases, there is only one propagator Green's function $G^{e}$ with $\log$ expansion $H^{e(s)}$ and it is given by $R^{-1 / s} .{ }^{20}$ The NNLL approximation can be obtained in two ways: either by inserting the relation between the $\beta$-function and the anomalous dimension into Eq. (2.20) or by using the explicit expression for the log expansion of the invariant charge in Eqs. $(2.11,2.15-2.17)$. In both cases, we obtain

$$
\begin{aligned}
H^{e(s)}(\alpha, z)=R_{0}(z)^{-1 / s}\left[1-\alpha \frac{\beta_{1} R_{0}(z)}{s \beta_{0}} \log R_{0}(z)+\alpha^{2} \frac{\beta_{1}^{2} R_{0}(z)^{2}}{s \beta_{0}^{2}}\right. \\
\left.\quad \times\left(\beta_{0} z\left(1-\frac{\beta_{0} \beta_{2}}{\beta_{1}^{2}}\right)-\log R_{0}(z)+\frac{1-s}{2 s} \log ^{2} R_{0}(z)\right)+\mathcal{O}\left(\alpha^{3}\right)\right] .
\end{aligned}
$$

For this class of toy models, equivalent expressions have been derived using chord diagrams in [10]. These formulas contain the symbols $a_{i, j}$, which are related to the anomalous dimension $\gamma^{e}$ as follows:

$$
\begin{aligned}
& \gamma_{0}^{e(1)}=-a_{1,0}, \quad \gamma_{1}^{e(1)}=-a_{2,0}, \quad \gamma_{2}^{e(1)}=-\left(a_{3,0}+a_{2,1} a_{1,0}\right), \\
& \gamma_{0}^{e(2)}=-a_{1,0}, \quad \gamma_{1}^{e(2)}=-\left(a_{2,0}+a_{1,1} a_{1,0}\right), \\
& \gamma_{2}^{e(2)}=-\left(a_{3,0}+a_{1,1} a_{2,0}+a_{1,1}^{2} a_{1,0}+3 a_{1,2} a_{1,0}^{2}+3 a_{2,1} a_{1,0}\right) .
\end{aligned}
$$

Using this translation, Eq. (2.22) agrees with the expressions found in [10].

20 Note that this is consistent with Eqs. $(2.13,2.18$ and 2.21). 


\section{Mathematical background}

This section collects the essential properties of Feynman graphs and renormalized Feynman rules that lead to the Callan-Symanzik equation (2.7). The derivation of this relation is given in Sect. 3.4 and requires two main ingredients: The first one is the well-known exponential formula for $\phi_{R}$; see Eq. (3.8). We motivate this property of renormalized Feynman rules and give a short introduction to the Hopf algebra of Feynman graphs in Sect. 3.2. The second ingredient is an (also well-known) expression for the coproduct of the infinite sums $X^{r}$ in Eqs. (1.5) and (1.6); see Eq. (3.10). We review in Sect. 3.3, how this relation is obtained from fixed-point equations for $X^{r}$, i.e., so-called combinatorial Dyson-Schwinger equations (DSEs). In the literature, the latter are written down in full detail only for those sums $X^{r}$ with $\phi_{R}(r)=1,{ }^{21}$ which is the reason why we restrict our results to that case. For presentation purposes, we consider QFTs with only one interaction term in the Lagrangian. (We explain the redefinition of the coupling constant in Sect. 3.1.) However, DSEs also exist in theories with more than one coupling parameter - the generalization to that case is given in Sect. 4.

\subsection{The grading of $\mathcal{H}$}

For QFTs with only one interaction term in the Lagrangian, one redefines the coupling constant as $\alpha=g^{l}$, which implies that a $K$-loop graph maps to a term proportional to $\alpha^{K}$ under renormalized Feynman rules. Let us show this now.

First, Euler's formula states that for a graph with $K$ loops, $V$ vertices and $E$ internal edges, the following identity holds:

$$
K+V-E=1 .
$$

Secondly, let $|v|$ be the degree of the vertex $v^{22}$ that corresponds to the interaction term in the Lagrangian. For example, $|v|=3$ in QED and $|v|=4$ in $\phi^{4}$ theory. Then, a Feynman graph with residue $r$ has $|r|$ external edges; hence,

$$
V \cdot|v|=2 E+|r|
$$

Together with Euler's formula and the definition of $l:=2 /(|v|-2)$, one finally finds

$$
V=l K+\left\{\begin{array}{ll}
0, & \text { for }|r|=2 \\
1, & \text { for }|r|=|v|
\end{array} .\right.
$$

One concludes that with the correct normalization of renormalized Feynman rules, a graph with $K$ loops and $V$ vertices maps to a term that is proportional to $g^{l K}=\alpha^{K}$.

${ }^{21}$ Hence, $|r|=2$ or $r=v$ is the vertex that corresponds to the interaction term in the Lagrangian.
22 That is, the number of edges which are incident to $v$. 


\subsection{Feynman rules from a Hopf algebraic point of view}

This section represents a minimal introduction to the Hopf algebra of Feynman graphs $[13,14]$. As a motivation, consider a typical observation during the renormalization of Feynman amplitudes: ${ }^{23}$

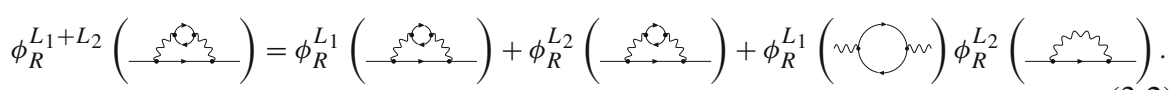

Here, and only within this section, we write renormalized Feynman rules $\phi_{R}^{L_{i}}$ with an upper index $L_{i}$. It indicates the energy scale $L_{i}=\log S_{i} / S_{0}$ at which Feynman graphs are evaluated. The goal of this section is to generalize the above observation (to the exponential formula). This can be achieved in a very elegant way, once we introduced the Hopf algebra of Feynman graphs.

Let us start with the algebra $\mathcal{H}$, i.e., the vector space $\mathcal{H}$ equipped with an associative product $m: \mathcal{H} \otimes \mathcal{H} \rightarrow \mathcal{H}$ (the disjoint union) and a unit element $\mathbb{I}$ (the empty graph). In a more mathematical notation,

$$
\begin{aligned}
\text { associativity: } & m \circ(m \otimes \mathrm{id})=m \circ(\mathrm{id} \otimes m), \\
\text { unit element: } & m(X \otimes \mathbb{I})=m(\mathbb{I} \otimes X)=X \quad \forall X \in \mathcal{H},
\end{aligned}
$$

where id denotes the identity map on $\mathcal{H}$.

In a first step, a coproduct $\Delta$ and a co-unit $\hat{\mathbb{I}}$ are defined, such that $\mathcal{H}$ extends to a bi-algebra. The important tool for our purposes is the coproduct. It is a linear map $\Delta: \mathcal{H} \rightarrow \mathcal{H} \otimes \mathcal{H}$, which decomposes a graph into its subgraphs in a certain way. The explicit definition of its action on 1PI Feynman graphs is given by

$$
\Delta \Gamma=\Gamma \otimes \mathbb{I}+\mathbb{I} \otimes \Gamma+\sum_{\gamma \subsetneq \Gamma} \gamma \otimes \Gamma / \gamma,
$$

where the sum extends over (disjoint unions of) divergent subgraphs $\gamma$ of $\Gamma$. Furthermore, $\Gamma / \gamma$ corresponds to $\Gamma$, but with the subgraphs $\gamma$ shrunk to a point. For example,

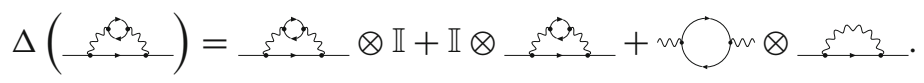

On a disjoint union of Feynman graphs, $\Delta$ is defined such that it is compatible with the product (a requirement for bi-algebras),

$$
\Delta(X Y)=\sum X^{\prime} Y^{\prime} \otimes X^{\prime \prime} Y^{\prime \prime}
$$

Here, Sweedler's notation for the coproduct is used, e.g., $\Delta X=\sum X^{\prime} \otimes X^{\prime \prime}$. The co-unit $\hat{\mathbb{I}}: \mathcal{H} \rightarrow \mathbb{K}$ maps an element $X \in \mathcal{H}$ to the coefficient of $\mathbb{I}$ in $X$. Indeed, it is

\footnotetext{
23 Note that this relation only makes sense because all scattering angles are set to $\boldsymbol{\Theta}_{0}$-in other words, $\phi_{R}$ is normalized appropriately.
} 
easy to show that the coproduct is co-associative and that $\hat{\mathbb{I}}$ is the co-unit element for $\Delta$

$$
\begin{array}{ll}
\text { co-associativity: } & (\Delta \otimes \mathrm{id}) \circ \Delta=(\mathrm{id} \otimes \Delta) \circ \Delta, \\
\text { co-unit element: } & (\hat{\mathbb{I}} \otimes \mathrm{id}) \circ \Delta=(\mathrm{id} \otimes \hat{\mathbb{I}}) \circ \Delta \simeq \mathrm{id} .
\end{array}
$$

Hence, $(\mathcal{H}, m, \mathbb{I}, \Delta, \hat{\mathbb{I}})$ forms a bi-algebra.

Secondly, one defines an antipode to further extend $\mathcal{H}$ to a Hopf algebra. It is the linear map $S: \mathcal{H} \rightarrow \mathcal{H}$, which is recursively given by

$$
m \circ(S \otimes \mathrm{id}) \circ \Delta=m \circ(\mathrm{id} \otimes S) \circ \Delta=\mathbb{I} \circ \hat{\mathbb{I}}, \quad S \circ m=m \circ(S \otimes S) .
$$

All in all, $(\mathcal{H}, m, \mathbb{I}, \Delta, \hat{\mathbb{I}}, S)$ forms a Hopf algebra, called the Hopf algebra of Feynman graphs.

With the above-defined structures, the vector space $\operatorname{Hom}(\mathcal{H}, \mathcal{A})$ of algebra homomorphisms extends to a group $G_{\mathcal{H}}^{\mathcal{A}}$, which is called the convolution or character group [23]. For example, renormalized Feynman rules are a character, $\phi_{R} \in G_{\mathcal{H}}^{\mathcal{A}}$. The conjunction in $G_{\mathcal{H}}^{\mathcal{A}}$ is the convolution product $\star$ : let $\phi, \psi \in G_{\mathcal{H}}^{\mathcal{A}}$. Then,

$$
\phi \star \psi:=m_{\mathcal{A}} \circ(\phi \otimes \psi) \circ \Delta
$$

is also an algebra homomorphism; hence, $\phi \star \psi \in G_{\mathcal{H}}^{\mathcal{A}}$. The convolution is associative because the coproduct is co-associative and the product $m_{\mathcal{A}}$ in $\mathcal{A}[\alpha, L]$ (multiplication) is associative. The unit element in $G_{\mathcal{H}}^{\mathcal{A}}$ is the linear map $e=1 \circ \hat{\mathbb{I}}$, and the inverse of a character $\phi$ is given by $\phi \circ S$.

We can now state a fundamental property of renormalized Feynman rules:

$$
\phi_{R}^{L_{1}+L_{2}}=\phi_{R}^{L_{1}} \star \phi_{R}^{L_{2}}
$$

This follows from a more general decomposition relation [2,3], which reduces to Eq. (3.7), because $\phi_{R}^{L}$ evaluates Feynman graphs for scattering angles at the renormalization point, $\boldsymbol{\Theta} \rightarrow \boldsymbol{\Theta}_{0}$. Note that Eq. (3.2) is a special case of Eq. (3.7).

The above relation implies that the vector space of maps $\phi_{R}^{L}$, equipped with the convolution product, forms a group. This is the so-called renormalization group and it is isomorphic to the additive group. Hence, there exists a linear map $\sigma: \mathcal{H} \rightarrow \mathcal{A}$, such that

$$
\phi_{R}=\exp ^{\star}(L \sigma)=e+L \sigma+\frac{L^{2}}{2} \sigma \star \sigma+\cdots
$$

This is the exponential formula (see, e.g., [2,3]). Here, we dropped the upper index in $\phi_{R}$ again, in order to adapt the notation to the rest of this paper.

The map $\sigma$ is an infinitesimal character, and we can give a meaning to it: it maps a (disjoint union of) Feynman graph(s) to the $L$-linear part of the corresponding Feynman amplitude, 


$$
\begin{aligned}
\sigma: \mathcal{H} & \rightarrow \mathcal{A} \\
X & \mapsto \sigma(X)=\left.\frac{\partial \phi_{R}(X)}{\partial L}\right|_{L=0}
\end{aligned}
$$

In particular, the infinite sums $X^{r}$ in Eqs. (1.5) and (1.6) are mapped to the negative of the anomalous dimension (see Eq. 2.4),

$$
\sigma\left(X^{r}\right)=-\gamma^{r}(\alpha)=-\sum_{k \geq 0} \gamma_{k}^{r} \alpha^{k+1} .
$$

From now on, we do not need the co-unit and antipode of the Hopf algebra anymore. These structures were only required for a proper definition of the convolution group $G_{\mathcal{H}}^{\mathcal{A}}$. The proof of Eq. (2.7) only uses the coproduct and the exponential formula in Eq. (3.8).

\subsection{Graph insertion and Dyson-Schwinger equations}

In this section, we motivate the following formula for the coproduct ${ }^{24}$ of the infinite sums $X^{r}$ in Eqs. (1.5) and (1.6): ${ }^{25}$

$$
\Delta X^{r}=\left.\sum_{k \geq 0} X^{r} X_{Q}^{k} \otimes X^{r}\right|_{k}
$$

Here, $X_{Q} \in \mathcal{H}$ is the combinatorial invariant charge $\left(\phi_{R}\left(X_{Q}\right)=Q\right)$, see Eq. (2.3). For example, in QED:

$$
X_{Q}=\frac{\left(X^{m \times}\right)^{2}}{X^{m}\left(X^{-}\right)^{2}} .
$$

Furthermore, $\left.\right|_{k}$ denotes the projection onto $\mathcal{H}_{k}$; hence, $\phi_{R}\left(\left.X\right|_{k}\right) \propto \alpha^{k}$ for each $X \in \mathcal{H}$.

Let us start by introducing the notion of graph insertion. Therefore, consider, for example, the QED Feynman graphs

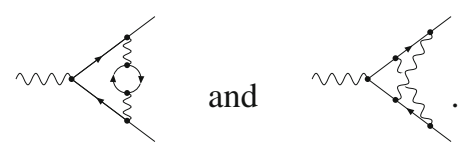

The first one is obtained, when the subgraph $m \sim$ is inserted into $m$. However, the second one does not contain any subgraph and cannot be constructed via graph insertion. Note that the coproduct of a 1PI Feynman graph $\Gamma$ without subgraphs is given by $\Delta \Gamma=\Gamma \otimes \mathbb{I}+\mathbb{I} \otimes \Gamma$; see Eq. (3.3). Hence, $\phi_{R}(\Gamma) \propto L$ by the exponential formula (3.8) and all graphs $\Gamma$ with that property are called primitive graphs. On

\footnotetext{
${ }^{24}$ This relation is well known in the literature; see, e.g., [24-27], or in a slightly different context, also [28,29]. Generalizations thereof can be found in [30,31].

${ }^{25}$ Note that $X^{r}$ maps to the Green's functions $G^{r}$ under renormalized Feynman rules.
} 
the other hand, each non-primitive 1PI Feynman graph can be obtained via graph insertions of primitive ones.

In the following, we make this more explicit. Therefore, let $\Gamma$ be a primitive graph. Each vertex of $\Gamma$ represents an insertion place for a 1PI vertex graph. Furthermore, each internal edge of type $e$ in $\Gamma$ is an insertion place for 1PI propagator graphs with residue $e$. Note that more than one propagator graph can be inserted into an edge, whereas only one vertex graph can be inserted into a vertex of $\Gamma$. Now, for each primitive $\Gamma$, define an insertion operator as a linear map $B_{+}^{\Gamma}: \mathcal{H} \rightarrow \mathcal{H}$, such that a disjoint union of 1PI Feynman graphs maps to

$$
B_{+}^{\Gamma}\left(\Gamma_{1} \cdots \Gamma_{n}\right)=\sum \frac{\tilde{\Gamma}}{\operatorname{sym}(\tilde{\Gamma})} .
$$

Here, the sum extends over all graphs $\tilde{\Gamma}$ that can be obtained by insertion of $\Gamma_{1}, \ldots, \Gamma_{n}$ into the various insertion places of $\Gamma$. The factor $\operatorname{sym}(\tilde{\Gamma})$ is assigned such that the insertion operator commutes with the coproduct in the following way: ${ }^{26}$

$$
\Delta B_{+}^{\Gamma}(\cdot)=B_{+}^{\Gamma}(\cdot) \otimes \mathbb{I}+\left(\mathrm{id} \otimes B_{+}^{\Gamma}\right) \Delta(\cdot) .
$$

The following examples should make Eq. (3.12) clear. For a more rigorous definition, see [15]:

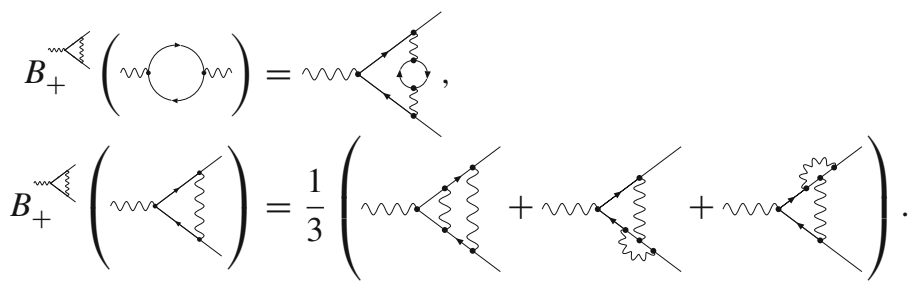

Note that by Eq. (3.3), these examples imply Eq. (3.13).

Some remarks are in order: First, inserting zero-loop graphs into $\Gamma$ does not change it. This is consistent with our realization of zero-loop graphs as the unit element $\mathbb{I} \in \mathcal{H}$. For example, $B_{+}^{\Gamma}(\mathbb{I})=\Gamma$ and $B_{+}^{\Gamma}\left(\Gamma_{1} \mathbb{I}\right)=B_{+}^{\Gamma}\left(\Gamma_{1}\right)$. Secondly, inserting more than $V$ vertex graphs into a primitive with $V$ vertices does not give a well-defined object in $\mathcal{H}$. For example, there is no element in $\mathcal{H}$ that corresponds to

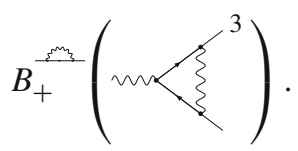

Finally, there are non-primitive graphs in $\mathcal{H}$ that are not in the image of some insertion operator. For example, $m \leqslant$ contains a subgraph, but it is not in the image of $B_{+}$. However, the infinite sums $X^{r}$ in Eqs. (1.5) and (1.6) consist of such linear combi-

26 The $B_{+}^{\Gamma}$ are Hochschild 1-co-cycles. 
nations of 1PI Feynman graphs that are in the image of some insertion operator $B_{+}^{\Gamma}$. This will be essential in the following.

The closed formula for the coproduct in Eq. (3.10) can be found from fixed-point relations (DSEs) for the infinite sums $X^{r}$. Let us motivate this again at the QED example, where the sums of all 1PI Feynman graphs of a certain residue satisfy [32]

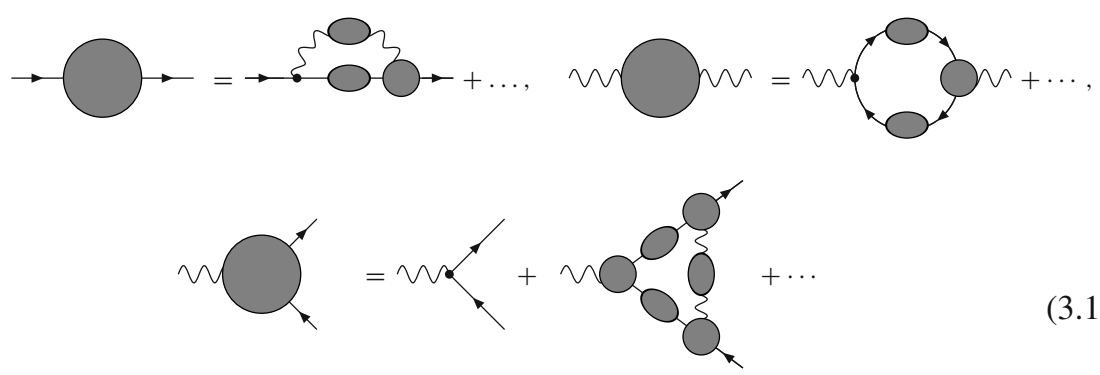

Here, we recall the notation of the introduction: A circle represents the sum of all respective 1PI graphs and an ellipse denotes the geometric series of propagator Feynman graphs, as in Eq. (1.4). ${ }^{27}$ On the rhs, these infinite sums are inserted into the one-loop primitives
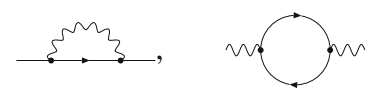

and

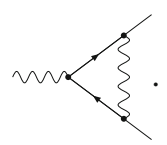

Here, only one vertex of the propagator primitives is dressed with an infinite sum in order to avoid double counting of graphs. The dots in Eq. (3.14) represent all graph insertions into higher loop primitives. The QED example is somewhat special, because the propagator DSE can be formulated for the quenched limit. Then, the first two fixed-point relations in Eq. (3.14) are actually complete.

Equation (3.14) translate to DSEs for the infinite sums $X^{r}$ in Eqs. (1.5) and (1.6). These can be written in a very elegant way using the insertion operators:

$$
\begin{aligned}
& X^{-}=\mathbb{I}-B_{+}^{+X_{3}^{m}}\left(\frac{\left(X^{m}\right)^{2}}{X^{m} X^{-}}\right), \quad X^{m}=\mathbb{I}-B_{+}^{m}\left(\frac{\left(X^{m}\right)^{2}}{\left(X^{-}\right)^{2}}\right), \\
& X^{m<}=\mathbb{I}+B_{+}^{m<}\left(\frac{\left(X^{m<}\right)^{3}}{X^{m}\left(X^{-}\right)^{2}}\right)+\cdots
\end{aligned}
$$

Note that there is no double counting of Feynman graphs, although the structure differs from the one in Eq. (3.14). Indeed, the (negative) power of $X^{-}\left(X^{e}\right)$ in the argument of an insertion operator $B_{+}^{\Gamma}$ corresponds to the number of vertices (type $e$ edges) in $\Gamma$. Hence, in contrast to Eq. (3.14), each insertion place is dressed by an infinite sum.

\footnotetext{
27 Remember that the zero-loop propagator graph is contained in the ellipse, but not in the circle on an edge-it belongs to the geometric series, but not to the sum of 1PI graphs.
} 
Here, the insertion operators take care that there is no double counting and the deeper reason for that is the commutation relation in Eq. (3.13) [15].

With these considerations, the above relations generalize in a simple way. Denote the sum of all insertion operators $B_{+}^{\Gamma}$ for $K$-loop primitives $\Gamma$ with residue $r$ by

$$
B_{+}^{K ; r}=\sum_{\substack{\Gamma \in \mathcal{H}_{K} \text { primitive } \\ \text { res }(\Gamma)=r}} B_{+}^{\Gamma} .
$$

Then, the general DSEs [11,12,29,32] for the infinite sums $X^{r}$ in Eqs. (1.5) and (1.6) with $\phi_{R}(r)=1 \mathrm{read}$

$$
X^{r}=\mathbb{I}+\operatorname{sgn}(r) \sum_{K \geq 1} B_{+}^{K ; r}\left(X^{r} X_{Q}^{K}\right) .
$$

Before we proceed, we quickly show that the insertion places of each $K$-loop primitive graph are fully dressed by infinite sums in Eq. (3.17): ${ }^{28}$ Consider a $K$-loop primitive graph $\Gamma$. The argument of the corresponding insertion operator $B_{+}^{\Gamma}$ is $X^{r} X_{Q}^{K}$, where by Eqs. (2.1) and (2.3),

$$
X_{Q}=\left(\frac{X^{v}}{\prod_{e \sim v} \sqrt{X^{e}}}\right)^{l} .
$$

The power of $X^{v}$ in the argument of $B_{+}^{\Gamma}$ is $l \cdot K$ for $|r|=2$ and $l \cdot K+1$, if $r=v$. This matches the number of vertices $V$ in $\Gamma$, given by Eq. (3.1). Secondly, the power of $1 / X^{e}$ in the argument of $B_{+}^{\Gamma}$ for some edge-type residue $e$ corresponds to

$$
l K \sum_{e \sim v} \frac{1}{2}-\left\{\begin{array}{ll}
1, & \text { if } r=e \\
0, & \text { else }
\end{array}=V \sum_{e \sim v} \frac{1}{2}- \begin{cases}1, & \text { if } r=e \\
\sum_{e \sim v} \frac{1}{2}, & \text { if } r=v \\
0, & \text { if }|r|=2, r \neq e\end{cases}\right.
$$

This equals the number of type $e$ internal edges in $\Gamma$, which completes the proof. Note that Eq. (3.15) coincide with the general form in Eq. (3.17), but only include the $K=1$ terms.

To close this section, we show that the coproduct formula in Eq. (3.10) and the commutation relation in Eq. (3.13) are consistent with the DSE in Eq. (3.17). With slightly more effort, one can turn this into a proof of Eq. (3.10) by induction. For details, see, e.g., [24-27]. First, let $M\left(X^{r}\right)=\prod_{r}\left(X^{r}\right)^{s_{r}}$ with integers $s_{r}$ be an arbitrary monomial in $X^{r}$. Then, Eq. (3.10) implies that

$$
\Delta M\left(X^{r}\right)=\left.\sum_{k \geq 0} M\left(X^{r}\right) X_{Q}^{k} \otimes M\left(X^{r}\right)\right|_{k} .
$$

\footnotetext{
$\overline{28}$ The reader may consider this as a motivation that Eq. (3.17) indeed holds. Note that the insertion operators ensure that there is no double counting. 
Now, we compute the coproduct of the rhs in Eq. (3.17). We commute $\Delta$ with the insertion operators via Eq. (3.13) and use the above relation for the monomials:

$$
\Delta[r h s(3.17)]=X^{r} \otimes \mathbb{I}+\operatorname{sgn}(r) \sum_{K \geq 1} \sum_{k \geq 0}\left(\mathrm{id} \otimes B_{+}^{K ; r}\right)\left(\left.X^{r} X_{Q}^{K+k} \otimes\left(X^{r} X_{Q}^{K}\right)\right|_{k}\right)
$$

Finally, we rearrange the sums as $\sum_{k \geq 1} \sum_{K=1}^{k}$ using an index shift, which results in

$$
\Delta[r h s(3.17)]=X^{r} \otimes \mathbb{I}+\sum_{k \geq 1}\left(X^{r} X_{Q}^{k} \otimes \operatorname{sgn}(r) \sum_{K=1}^{k} B_{+}^{K ; r}\left(\left.X^{r} X_{Q}^{K}\right|_{k-K}\right)\right) .
$$

Note that the terms on the rhs of the $\otimes$ sign correspond to $\left.X^{r}\right|_{k}$ (see Eq. 3.17). Hence, we are left with

$$
\Delta[r h s(3.17)]=X^{r} \otimes \mathbb{I}+\left.\sum_{k \geq 1} X^{r} X_{Q}^{k} \otimes X^{r}\right|_{k}=\left.\sum_{k \geq 0} X^{r} X_{Q}^{k} \otimes X^{r}\right|_{k}=\Delta[\operatorname{lhs}(3.17)]
$$

\subsection{Rederivation of the Callan-Symanzik equation}

With the considerations of the previous sections, the proof of Eq. (2.7) is simple. It requires the exponential formula (3.8) and the coproduct relation (3.10). Together with the definitions of the convolution product in Eq. (3.6) and the $\sigma$ function in Eq. (3.9), we find

$$
\begin{aligned}
\frac{\partial G^{r}}{\partial L} & =\frac{\partial}{\partial L} \phi_{R}\left(X^{r}\right)=\frac{\partial}{\partial L} \exp ^{\star}(L \sigma)\left(X^{r}\right)=\left(\phi_{R} \star \sigma\right)\left(X^{r}\right) \\
& =\sum_{k \geq 0} \phi_{R}\left(X^{r} X_{Q}^{k}\right) \sigma\left(\left.X^{r}\right|_{k}\right)=\sum_{k \geq 1} G^{r} Q^{k}\left(-\gamma_{k-1}^{r} \alpha^{k}\right)=-G^{r} \gamma^{r}(\alpha Q) .
\end{aligned}
$$

\section{Multiple coupling constants}

In this section, we rederive the Callan-Symanzik equation from Dyson-Schwinger equations in QFTs with multiple interaction terms in the Lagrangian. We assume that each such term comes with a separate coupling constant and gives rise to a certain vertex in Feynman graphs. We denote the different vertices by $v_{1}, \ldots, v_{m}$ and collect the coupling parameters to a vector $g=\left(g_{1}, \ldots, g_{m}\right)$. The positive integer $m$ is given by the number of interaction terms. In the following, we summarize the main changes to the one coupling case.

First of all, it is inappropriate to grade the Hopf algebra $\mathcal{H}$ of Feynman graphs with respect to the loop number in this setting. Instead, $\mathcal{H}$ is multi-graded as $[14,18,30,33]$

$$
\mathcal{H}=\bigoplus_{\mathbf{k}} \mathcal{H}_{\mathbf{k}}
$$


Here, $\mathbf{k}=\left(k_{1}, \ldots, k_{m}\right)$ denotes a multi-index (a vector of integers) and the subspaces $\mathcal{H}_{\mathbf{k}}$ contain the following Feynman graphs:

$$
\Gamma \in \mathcal{H}_{\mathbf{k}} \Leftrightarrow \Gamma \text { has }\left\{\begin{array}{ll}
k_{j} \text { vertices } v_{j}, & \text { if }|\operatorname{res} \Gamma|=2 \\
k_{j}+\delta_{i j} \text { vertices } v_{j}, & \text { if } \operatorname{res} \Gamma=v_{i}
\end{array} .\right.
$$

Note that this grading respects the product and coproduct of $\mathcal{H}$.

Secondly, the Green's functions $G^{r}$ depend on all coupling constants $g$ and the logarithmic energy scale $L$. In particular, $G^{r}(g, L)=\phi_{R}\left(X^{r}\right)$ as before, where the $X^{r}$ are given by the infinite sums in Eqs. (1.5) and (1.6). Hence, the corresponding anomalous dimensions $\gamma^{r}$ are power series in all coupling parameters,

$$
\gamma^{r}(g)=-\left.\frac{\partial G^{r}(g, L)}{\partial L}\right|_{L=0}=\sum_{\mathbf{k}} \gamma_{\mathbf{k}}^{r} g_{1}^{k_{1}} \ldots g_{m}^{k_{m}} .
$$

Furthermore, there is one amputated Green's function (invariant charge) for each vertex $v_{i}$; see Eq. (2.1). These define the running coupling parameters as before. In order to simplify the notation, we write

$$
X_{i}:=X_{\mathrm{amp}}^{v_{i}}, \quad Q_{i}(g, L):=\phi_{R}\left(X_{i}\right), \quad \tilde{g}=\left(\tilde{g}_{1}, \ldots, \tilde{g}_{m}\right)=\left(g_{1} Q_{1}, \ldots, g_{m} Q_{m}\right)
$$

Accordingly, there is one $\beta$-function for each vertex $v_{i}$, with power series expansion

$$
\beta^{i}(g)=\left.\frac{\partial Q_{i}(g, L)}{\partial L}\right|_{L=0}=\sum_{\mathbf{k}} \beta_{\mathbf{k}}^{i} g_{1}^{k_{1}} \cdots g_{m}^{k_{m}} .
$$

Finally, the Dyson-Schwinger equation for the infinite sums in Eqs. (1.5) and (1.6) is given by

$$
X^{r}=\mathbb{I}+\operatorname{sgn}(r) \sum_{\mathbf{k}} B_{+}^{\mathbf{k} ; r}\left(X^{r} X_{1}^{k_{1}} \cdots X_{m}^{k_{m}}\right),
$$

where

$$
B_{+}^{\mathbf{k} ; r}=\sum_{\substack{\Gamma \in \mathcal{H}_{\mathbf{k}} \text { primitive } \\ \operatorname{res}(\Gamma)=r}} B_{+}^{\Gamma}
$$

is the generalization of Eq. (3.16); see, e.g., [18,34]. Note that in Eq. (4.1), each insertion place of a primitive $\Gamma$ is fully dressed with an infinite sum and there is no double counting of graphs because the insertion operators $B_{+}^{\mathbf{k} ; r}$ are Hochschild 1-cocycles, i.e., they satisfy the commutation relation in Eq. (3.13). In full analogy to the end of Sect. 3.3, the DSE in Eq. (4.1) implies the coproduct formula (see, e.g., [30,31])

$$
\Delta X^{r}=\left.\sum_{\mathbf{k}} X^{r} X_{1}^{k_{1}} \cdots X_{m}^{k_{m}} \otimes X^{r}\right|_{\mathbf{k}}
$$

where $\left.\right|_{\mathbf{k}}$ is the projection onto $\mathcal{H}_{\mathbf{k}}$. This is the generalization of Eq. (3.10). 
The Callan-Symanzik equation can now be derived in the same way as in Sect. 3.4. Therefore, the coproduct formula in Eq. (3.10) is replaced by Eq. (4.2) and the action of $\sigma$ on the infinite sums $X^{r}$ in Eq. (3.9) must be generalized to

$$
\sigma\left(\left.X^{r}\right|_{\mathbf{k}}\right)=-\gamma_{\mathbf{k}}^{r} g_{1}^{k_{1}} \cdots g_{m}^{k_{m}}
$$

Then, using the exponential formula in Eq. (3.8) and the definition of the convolution product in Eq. (3.6) results in

$$
\frac{\partial G^{r}}{\partial L}=\left(\phi_{R} \star \sigma\right)\left(X^{r}\right)=G^{r} \sum_{\mathbf{k}} Q_{1}^{k_{1}} \ldots Q_{m}^{k_{m}} \sigma\left(\left.X^{r}\right|_{\mathbf{k}}\right)=-G^{r} \gamma^{r}(\tilde{g}),
$$

which can be written in the same form as in the one coupling case; see Eq. (2.7):

$$
\frac{\partial}{\partial L} \log G^{r}(g, L)=-\gamma^{r}(\tilde{g}), \quad \tilde{g}=\left(g_{1} Q_{1}, \ldots, g_{m} Q_{m}\right) .
$$

In particular, taking certain linear combinations of these relations yields a system of ordinary differential equations, which is the analogue to Eq. (2.8),

$$
\frac{\partial}{\partial L} \log Q_{i}(g, L)=\beta^{i}(\tilde{g}), \quad \tilde{g}=\left(g_{1} Q_{1}, \ldots, g_{m} Q_{m}\right) .
$$

Together with the initial conditions $G^{r}(g, 0)=1$ and $Q_{i}(g, 0)=1$, Eqs. (4.3) and (4.4) fully determine the Green's functions, when only the anomalous dimensions and $\beta$-functions are known.

Two remarks are in order. First, in terms of the running coupling parameters, Eq. (4.4) represents the usual system of renormalization group equations ${ }^{29}$ and Eq. (4.3) is the usual form of the Callan-Symanzik equation for the Green's functions $\tilde{G}^{r}$ with $\tilde{G}^{r}(\tilde{g}, L)=G^{r}(g, L)$ :

$$
\frac{\partial \tilde{g}_{i}}{\partial L}=\tilde{g}_{i} \beta^{i}(\tilde{g}), \quad\left(\frac{\partial}{\partial L}+\sum_{i} \tilde{g}_{i} \beta^{i}(\tilde{g}) \frac{\partial}{\partial \tilde{g}_{i}}+\gamma^{r}(\tilde{g})\right) \tilde{G}^{r}(\tilde{g}, L)=0 .
$$

Secondly, the log expansions for the Green's functions can be derived as shown in Sect. 2.2 via a change of variables. Here, the main difference to the previous case is that we have several coupling constants, but only one energy scale. Hence, one has to choose one coupling $g_{i}$ and changes $L \rightarrow z=g_{i} L$. In particular, let $G^{r}(g, L)=H^{r}\left(g, g_{i} L\right)$. Then, the solution to Eq. (4.3) is given by

$$
H^{r}(g, z)=\exp \left(-\int_{0}^{z} \frac{\gamma^{r}(\tilde{g})}{g_{i}} \mathrm{~d} z^{\prime}\right)
$$

This formula only requires the log expansion for the running coupling parameters $\tilde{g}$, which can be found log order by log order from the RGE. Note that the resulting log

29 Again, our definition of the $\beta$-functions differs from the one in the literature, $\beta_{\text {lit }}^{i}(\tilde{g})=\tilde{g}_{i} \beta^{i}(\tilde{g})$. 
expansion will depend on the choice of $g_{i}$. We hope to investigate such cases further and give examples in future work.

\section{Conclusions}

In this paper, we established a precise connection between Dyson-Schwinger equations for the Green's functions in a given QFT and their corresponding log expansions. We mainly discussed QFTs with only one interaction term in the Lagrangian (Sect. 2.2), but also expanded the results to the more general case (Sect. 4).

The formulas for the log expansions are given in Eqs. (2.18) and (4.5) - the nextto ${ }^{(n)}$-leading log approximation is obtained by an analytical integration that requires the solution of the renormalization group equation up to the next-to ${ }^{(n)}$-leading $\log$ order. In particular, it only depends on the $(n+1)$-loop $\beta$-function and anomalous dimension. An explicit expression for the next-to-next-to-leading log approximation is given in Eq. (2.20), when only one coupling constant is involved. Restricting to special cases, our formulas reproduce the known expressions in the literature. These cases include the photon propagator Green's function in quantum electrodynamics and a toy model, where all Feynman graphs with vertex sub-divergences are neglected. Compare Eq. (2.19) with [9] and Eqs. (2.22, 2.23) with [10].

Our results may be used for an accurate perturbative calculation of the Green's functions, for energy scales far away from the renormalization scale $S \gg S_{0}$. However, the log expansions do only converge, if the coefficients do well behave perturbatively. The latter must not be true. So far, we have no control about the dependence of the Green's functions on the scattering angles $\boldsymbol{\Theta}_{0}$ at the renormalization point. In order to understand this better, one needs to generalize the exponential formula in Eq. (3.8) to include also the angle dependence. Together with the coproduct formulas in Eqs. (3.10) and (4.2), this would lead to a generalization of the Callan-Symanzik equation, from which the correct perturbative approach may be obtained. However, this is a highly non-trivial problem, as it requires to understand the behavior of the Green's functions orthogonal to the renormalization group flow.

Our work gives the derivation of the log expansions for the Green's functions from the corresponding Dyson-Schwinger equations. However, if the latter are not known, we cannot comment on the corresponding log expansions. For example, in QED, a detailed Dyson-Schwinger equation for the 4-point function $G^{X}$ in terms of the insertion operators is not explicitly written down in the literature. From there, one could generalize the coproduct formula in Eq. (3.10). Together with Eq. (2.7), this would lead to the respective Callan-Symanzik equation and then to the corresponding $\log$ expansion.

Finally, we would like to investigate the case with multiple coupling parameters. Here, we need to solve the system of renormalization group equations in Eq. (4.4) analytically, such that Eq. (4.5) can be integrated log order by log order.

Acknowledgements Open access funding provided by University of Vienna. First of all, I want to thank Dirk Kreimer and Karen Yeats for very illuminating discussions and for their patience in answering so many questions. Furthermore, I would like to thank Simon Plätzer and Angelika Widl for giving me insights to 
the particle physicist point of view. Finally, I acknowledge useful discussions with Henry Kißler, David Prinz, Stefan Fredenhagen, Michael Borinsky, John Gracey and Erik Panzer.

\section{Compliance with ethical standards}

Conflict of interest The author declares that there is no conflict of interest.

Open Access This article is licensed under a Creative Commons Attribution 4.0 International License, which permits use, sharing, adaptation, distribution and reproduction in any medium or format, as long as you give appropriate credit to the original author(s) and the source, provide a link to the Creative Commons licence, and indicate if changes were made. The images or other third party material in this article are included in the article's Creative Commons licence, unless indicated otherwise in a credit line to the material. If material is not included in the article's Creative Commons licence and your intended use is not permitted by statutory regulation or exceeds the permitted use, you will need to obtain permission directly from the copyright holder. To view a copy of this licence, visit http://creativecommons.org/licenses/by/4.0/.

\section{References}

1. Kißler, H.: On the gauge dependence of quantum electrodynamics. PoS (2018). https://doi.org/10. 22323/1.303.0032

2. Brown, F., Kreimer, D.: Angles, scales and parametric renormalization. Lett. Math. Phys. 103, 9331007 (2013). https://doi.org/10.1007/s11005-013-0625-6

3. Kreimer, D., Panzer, E.: Renormalization and Mellin transforms. In: Computer Algebra in Quantum Field Theory. Integration, Summation and Special Functions. Proceedings, LHCPhenoNet School: Linz, Austria, 9-13 July 2012, pp. 195-223 (2013). https://doi.org/10.1007/978-3-7091-1616-6_8. arXiv: 1207.6321

4. Peskin, M.E., Schroeder, D.V.: An Introduction to Quantum Field Theory. Addison-Wesley, Reading (1995)

5. Ward, J.C.: An identity in quantum electrodynamics. Phys. Rev. 78, 182 (1950). https://doi.org/10. 1103/PhysRev.78.182

6. Green, H.S.: A pre-renormalized quantum electrodynamics. Proc. Phys. Soc. A 66, 873-880 (1953). https://doi.org/10.1088/0370-1298/66/10/303

7. Takahashi, Y.: On the generalized ward identity. Nuovo. Cim. 6, 371 (1957). https://doi.org/10.1007/ BF02832514

8. Krüger, O., Kreimer, D.: Filtrations in Dyson-Schwinger equations: next-to ${ }^{j}$-leading log expansions systematically. Ann. Phys. 360, 293-340 (2015). https://doi.org/10.1016/j.aop.2015.05.013

9. Delage, L.: Leading log expansion of combinatorial Dyson-Schwinger equations. arXiv:1602.08705

10. Courtiel, J., Yeats, K.: Next-to ${ }^{k}$ leading log expansions by chord diagrams. arXiv:1906.05139

11. Kreimer, D., Yeats, K.: An Etude in non-linear Dyson-Schwinger equations. Nucl. Phys. Proc. Suppl. 160, 116-121 (2006). https://doi.org/10.1016/j.nuclphysbps.2006.09.036

12. Kreimer, D., Yeats, K.: Recursion and growth estimates in renormalizable quantum field theory. Commun. Math. Phys. 279, 401-427 (2008). https://doi.org/10.1007/s00220-008-0431-7

13. Kreimer, D.: On the Hopf algebra structure of perturbative quantum field theories. Adv. Theor. Math. Phys. 2, 303-334 (1998). https://doi.org/10.4310/ATMP.1998.v2.n2.a4

14. Connes, A., Kreimer, D.: Renormalization in quantum field theory and the Riemann-Hilbert problem. I. The Hopf algebra structure of graphs and the main theorem. Commun. Math. Phys. 210, 249-273 (2000). https://doi.org/10.1007/s002200050779

15. Kreimer, D.: Anatomy of a gauge theory. Ann. Phys. 321, 2757-2781 (2006). https://doi.org/10.1016/ j.aop.2006.01.004

16. van Suijlekom, W.D.: The structure of renormalization Hopf algebras for gauge theories. I: Representing Feynman graphs on BV-algebras. Commun. Math. Phys. 290, 291-319 (2009). https://doi.org/10.1007/ s00220-009-0829-x

17. Kreimer, D., Sars, M., van Suijlekom, W.D.: Quantization of gauge fields, graph polynomials and graph homology. Ann. Phys. 336, 180-222 (2013). https://doi.org/10.1016/j.aop.2013.04.019

18. Foissy, L.: Mulitgraded Dyson-Schwinger systems. arXiv:1511.06859 
19. Chetyrkin, K.G., Kuhn, J.H., Steinhauser, M.: RunDec: a mathematica package for running and decoupling of the strong coupling and quark masses. Comput. Phys. Commun. 133, 43-65 (2000). https:// doi.org/10.1016/S0010-4655(00)00155-7

20. Kißler, H.: Systems of linear Dyson-Schwinger equations

21. Ruijl, B., Ueda, T., Vermaseren, J.: FORM version 4.2. arXiv:1707.06453

22. van Suijlekom, W.: The Hopf algebra of Feynman graphs in QED. Lett. Math. Phys. 77, 265-281 (2006). https://doi.org/10.1007/s11005-006-0092-4

23. Connes, A., Kreimer, D.: Renormalization in quantum field theory and the Riemann-Hilbert problem. II. The beta function, diffeomorphisms and the renormalization group. Commun. Math. Phys. 216, 215-241 (2001). https://doi.org/10.1007/PL00005547

24. Yeats, K.A.: Growth estimates for Dyson-Schwinger equations. PhD thesis, Boston University, Massachusetts, (2008). arXiv:0810.2249

25. van Suijlekom, W.D.: Renormalization of gauge fields using Hopf algebras. In: Quantum Field Theory: Competitive Models. Proceedings, 3rd Workshop on Recent developements in quantum field theory, Leipzig, Germany, July 20-22, 2007, pp. 137-154 (2008). https://doi.org/10.1007/978-3-7643-87365_8. arXiv:0801.3170

26. Borinsky, M.: Feynman graph generation and calculations in the Hopf algebra of Feynman graphs. Comput. Phys. Commun. 185, 3317-3330 (2014). https://doi.org/10.1016/j.cpc.2014.07.023

27. Kißler, H.: Hopf-algebraic Renormalization of QED in the linear covariant Gauge. Ann. Phys. 372, 159-174 (2016). https://doi.org/10.1016/j.aop.2016.05.008

28. Bergbauer, C., Kreimer, D.: Hopf algebras in renormalization theory: locality and Dyson-Schwinger equations from Hochschild cohomology. IRMA Lect. Math. Theor. Phys. 10, 133-164 (2006). https:// doi.org/10.4171/028-1/4

29. Foissy, L.: Faà di bruno subalgebras of the hopf algebra of planar trees from combinatorial DysonSchwinger equations. Adv. Math. 218, 136-162 (2008)

30. Prinz, D.: Gauge symmetries and renormalization. arXiv:2001.00104

31. Kißler, H.: Computational and diagrammatic techniques for perturbative quantum electrodynamics. PhD thesis, Humboldt Universität zu Berlin, Berlin (2017)

32. Kreimer, D., van Suijlekom, W.D.: Recursive relations in the core Hopf algebra. Nucl. Phys. B 820, 682-693 (2009). https://doi.org/10.1016/j.nuclphysb.2009.04.025

33. Prinz, D.: Algebraic structures in the coupling of gravity to Gauge theories. arXiv:1812.09919

34. Rotheray, L.: Hopf subalgebras from green's functions. Master's thesis, Humboldt-Universität zu Berlin (2014)

Publisher's Note Springer Nature remains neutral with regard to jurisdictional claims in published maps and institutional affiliations. 